\title{
The Analysis of Network Public Opinion Monitoring Management System Based on B/S Architecture
}

\author{
Li Yan* \\ College of Information Engineering, Gansu Institute of Political Science and law, Lanzhou 730070, China
}

\begin{abstract}
Public opinion monitoring system takes advantage of the search engine technology and the network information mining technology, through the contents of the Web page such as automatic acquisition and processing, sensitive word filtration, intelligent clustering and classification, topic detection, thematic focus, statistical analysis. For these contents, every unit needs to be under the network of public opinion supervision and management system and its practical value is eventually formed as a result of public opinion presentation, public opinion, the special report, analysis report, and decision layer to have a comprehensive grasp of public opinion trends, which provides a correct guidance for the public opinion measurement, and supplies the basis for the analysis. A public opinion monitoring system has been introduced in this paper, which is based on the dynamic kernel with the structure of $\mathrm{B} / \mathrm{S}$ for the demonstration in the form of the software system. Combined with the actual market demand, it works through the effective technology of theoretical medicine and the implementation of a professional development team so as to ultimately achieve the goal of monitoring and control system of public opinion.
\end{abstract}

Keywords: Network public opinion, monitoring management system, B/S architecture.

\section{INTRODUCTION}

With a large number of public opinions flooding the network, and the help of the Internet high-speed transmission, public opinions have become a very powerful force for the government, companies, individuals etc. Artificial intelligence is the traditional way to better understand public opinion shown by passively browsing the Web sites, forums [1], and understanding public opinion, which result in a waste of a lot of manpower and time, and often in public opinion occurring long after learned. This misses the best PR process and the best window to be used. This paper from the actual project of using automation software monitoring system to monitor the implementation of network public opinion, through the actual software technology to implement the system, helps the user for the first time to master public opinion trends, which are not only public opinion on the nose [2], but also always take the initiative. At present, there are several kinds of public opinion monitoring systems on the market, which have different characteristics [3], but there are essential differences between the systems. This paper summarizes the previous research results, and through the relevant investigation of a large number of the user's actual needs, the design model and puts forward a different form of the traditional public opinion monitoring system, so as to achieve satisfactory results, as far as possible to meet the requirements of different users, and to ensure the system is easy to use.

*Address correspondence to this author at the Åbo Akademi University, P.O. Box 311, FIN-65101 Vasa, Finland; Tel: +358-6-3247476; Fax: +3586-3247457; E-mails: jan.saarela@abo.fi, fjalar.finnas@abo.fi
Therefore, in today's rapid development of information technology, the introduction of various technologies enable people to deal with a wider range of business and information, such as scheduling, information release, document flow, meeting organization, etc. Along with the network communication and distribution processing, the development of advanced technology such as database, network resource sharing and collaborative work makes the network office automation system to become a reality. Network public opinion monitoring is one of the most important news propaganda works, especially one of the key works of the government's propaganda and deployment; it is the most important tasks of the construction of network public opinion management system. At present, China's network public opinion management informatization system construction has entered a rapid advanced stage, and the use of modern information technology to promote the healthy and orderly development of the network public opinion information has become inevitable. And how the innovation mechanism, guarantees information security, to better promote the construction of public opinion management, and the healthy development of the network problems, becomes the governments need to face the important news propaganda department at all levels.

$\mathrm{B} / \mathrm{S}$ (Browser/Server) structure is emerged with the rise of the Internet technology, whereas the $\mathrm{C} / \mathrm{S}$ structure is a changed or improved structure. Working under this structure, the user interface is implemented via the WWW Browser, few transaction logics in the front-end (Browser), and the main transaction logic on the Server side (Server), forming a so-called three-layered structure. So it greatly simplifies the client computer load, reduces the cost of system maintenance and upgrades the workload, reducing the overall expense of users $[4,5]$. The three-layered diagram of $\mathrm{B} / \mathrm{S}$ architecture model structure is shown in Fig. (1). 


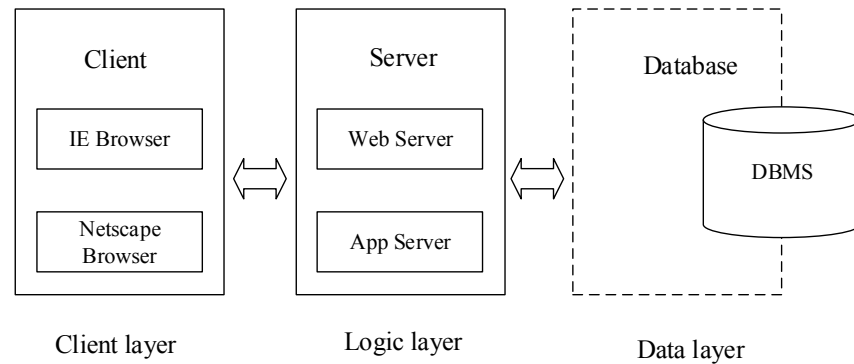

Fig. (1). The three layers of $B / S$ architecture.

Its main advantages are as follows [6-8]:

1. Good flexibility and extensibility. In the specific environment and practical applications, the conditions often change, so the system should be flexible, that is, as long as the application layer is to achieve, the corresponding changes will be able to reflect the changes in the system.

2. Shareable. A single application server can provide service for customer application at different platforms, in large part to save the development time and money.

3. Security. In this kind of structure, client applications can't directly access data. The application server can not only control what data has been changed and is accessed, but also can control the change of the data and the access method.

4. The enhancement enterprise repeating usability of objects. With the development of component technology, the reusable component model is more and more accepted for the software development.

5. Three layer model has to become in the true sense of "thin client", which has high stability, ductility and execution rate of the school. Three layer models can provide unified service management to the client, thus it has the good ability of fault tolerance and load balancing.

$\mathrm{B} / \mathrm{S}$ structure has the biggest advantage as it can be operated anywhere without having to install any special software. As long as there is a computer, it can be connected to the Internet and can be used, that is it requires the client's zero maintenance. The expansion of the system is very easy. As long as one can get to the Internet, the system can be used by assigning a user name and password by the system administrator.

\section{TOPIC DETECTION AND TRACKING TOOL}

Sensitive word list and degree. Based on the sensitive words that public opinion experts provide, we built a sensitive word list, and then set the degree for each sensitive word (according to its importance, sensitive degree); With TF-IDF (Term Frequency-Inverse Document Frequency) method, we transferred the text into vectors. Calculation formula is shown as follows:

$w_{i j}=t f_{i j} \times i d f_{i}$

$w_{i j}$ stands for the weight of word i in file j. $t f_{i j}$ stands for the frequency of word $\mathrm{i}$ in file $\mathrm{j}$. $i d f_{i}$ stands for the reciprocal of the file frequency of word $i$.
To strengthen the importance of a keyword in critical location, we enhanced the weight of keywords that appear in head, title, and first-level title of that Web page.

Cluster. We adopted the method of CMU proposed to cluster the vectors [2]. First, we calculated the similarity degree between every two vectors, for which we adopted cosine formula to calculate the similarity degree:

$\sin (x, c)=\frac{\sum_{j=1}^{M} w_{j x} \times w_{j c}}{\sqrt{\left(\sum_{j=1}^{M} w_{j x}^{2}\right) \times\left(\sum_{j=1}^{M} w_{j x}^{2}\right)}}$

$\sin (x, c)$ stands for the similarity degree between the vector $\mathrm{x}$, which comes from a new text and a cluster $\mathrm{c} ; w_{j c}$ stands for the weight of word $\mathrm{j}$ in the cluster $\mathrm{c} ; \mathrm{M}$ stands for total amount of words in the vector space. We adopted the kmeans method to cluster the vectors.

Topic Detection. Each cluster is viewed as an event, the average weight of which is then calculated. Firstly, we calculated the similarity degree between the vector coming from a new Web page and the existing average vector. Considering the importance of an event diminishing with the elapsing time, the same key word may have a completely different meaning, so we adopted the timespan calculation method. The calculation formula is shown as below:

$\operatorname{score}(x)=1-\max \left\{\left(1-\frac{k}{m}\right) \times \operatorname{sim}\left(\underset{x}{\vec{x}}, \overrightarrow{c_{i}}\right)\right\}$

In this formula, $\mathrm{x}$ stands for a new document vector; $c_{i}$ stands for the number $i$ cluster in the time area; I stands for the total amount of vectors in the vector space; $\mathrm{k}$ stands for the number of added document vectors coming between $\mathrm{x}$ and the latest one. If the outcome score is greater than the default value, the new file is viewed as a new topic.

\section{DEMAND ANALYSIS OF PUBLIC OPINION MON- ITORING SYSTEM}

All over the major search engines are now based on the feedback by providing vast amounts of data corresponding to the user's search request. Certain users pay no real attention to public opinion information, not even for their own calculation of information datasets processing, integration, analysis and judgment. Therefore, the system design cannot provide feedback to the users by providing initiative for public opinion information (now the service is passive). Of course, not to mention, combining the public opinion and the specific task of monitoring service for users provides a comprehensive range of system counterpart [9]. And the emergence of public opinion monitoring system can greatly make up for the shortcomings and disadvantages of the Internet search engine, because public opinion monitoring system can provide users with the initiative of the user's demand for services in addition to the public opinion information distance dataset. Also for the special requirements of users, the system can be generated to judge the trend of public opinion. Public opinion statements provide a decision aid, and so on. And these works by the public opinion system will greatly improve the working efficiency of the user, by allowing the users to timely, accurately and quickly find and grasp the key public opinion from the vast information available on the 
Internet. The module structure design for the public opinion monitoring system is shown in Fig. (2).

From the aspects of system function, data provided by the public opinion system is based on the rich statistical analysis. After filtration, screening, analysis and mining of statistical data, this kind of system mainly provides functions including, automatically discovering, trend analysis, project tracking, automatic alarming, automatic classification, etc. Besides it can also provide public opinion letter, the basic retrieval function of interest rates, and, in our daily life as is known, the Internet search engine in general, can also provide the basic information retrieval (or information search services), based on some commercial interests and political sensitivity, often because of a lot of irrational factors that can meet the needs of users. The range of public opinion class system adopted a set of information that is relatively narrow, such as sometimes it is only the basic orientation, or generally it is the system's users to concern with specific areas of information, or specific system areas of some of the Web sites can do comprehensive and accurate information acquisition and fetching.

Network public opinion management system through the information collection, text mining technology, such as social public opinion, can quickly find and collect the information, and carries on the automatic acquisition, intelligent filtering, automatic topic detection, automatic classification, clustering and statistical analysis. Whether it is the major cases happened in the society, emergency or social hot topic, the fast orientation tracking and recognition will help the government build a positive network environment. Public opinion obtained through the network information management, correctly guides the government departments through the control and management of the information toward public opinion propaganda and the development, thus providing a fast and effective channel. Keeping in this background, the design and research of network public opinion management system is based on $\mathrm{B} / \mathrm{S}$ mode. In order to analyze and track important hot news information, Internet public opinion management system can grasp the public opinion breaking point and the situation for the incident happened. System will provide hot news at different times [7], according to the community chain of transmission, and automatic tracking of the amount of news articles on each big Web site. Generally speaking, in a certain period of time, the number of news media sites growth can also reflect the growth of the chain of news propagation. Besides, it also provides hot posts, special reports, and other functions.

This system adopts the now widely popular B/S structure design, in which the transaction logic, data, information and process controls are placed on the Server side, whereas implementation and users need are placed on Browser client, the only mean through which the client can realize all the system both before and after the software operations and access. Part of this system has been divided into eight major subsystems, and each subsystem contains several submodules. Due to the limitation of space, this article will omit some subsystems or modules. As stated earlier, this system adopts B/S structure, namely the Browser/Server architecture model, the Browser client and Server side. Browser side is responsible for public opinion information display and basic interaction with users, the Server end is responsible for negative public opinion information and data processing and response to user actions and execution, namely the realization of the business logic and data information support. The system's overall architecture is shown in Fig. (3).

Because this system adopts the classic B/S structure, so the user reaches through the network connection to the system; this system uses a Web browser and App. All user interaction completes within the system through a Web browser. The system greatly simplifies the complexity of the user operations, improves the user experience, and lets users to quickly and conveniently use this system. At the same time, using B/S architecture can achieve such an effect of Internet surveillance of network public opinion through this system. For the same set of cases, implementing this system, no matter how many users, or how these users are geographically distributed, as long as the user can reach through the Web Browser on the Internet, can realize the operation and maintenance. In this way, users need to install the client prior on your computer, with any Web browser to access this system that can be found on the computer. This system is a set of software and hardware combined with integrated infor-

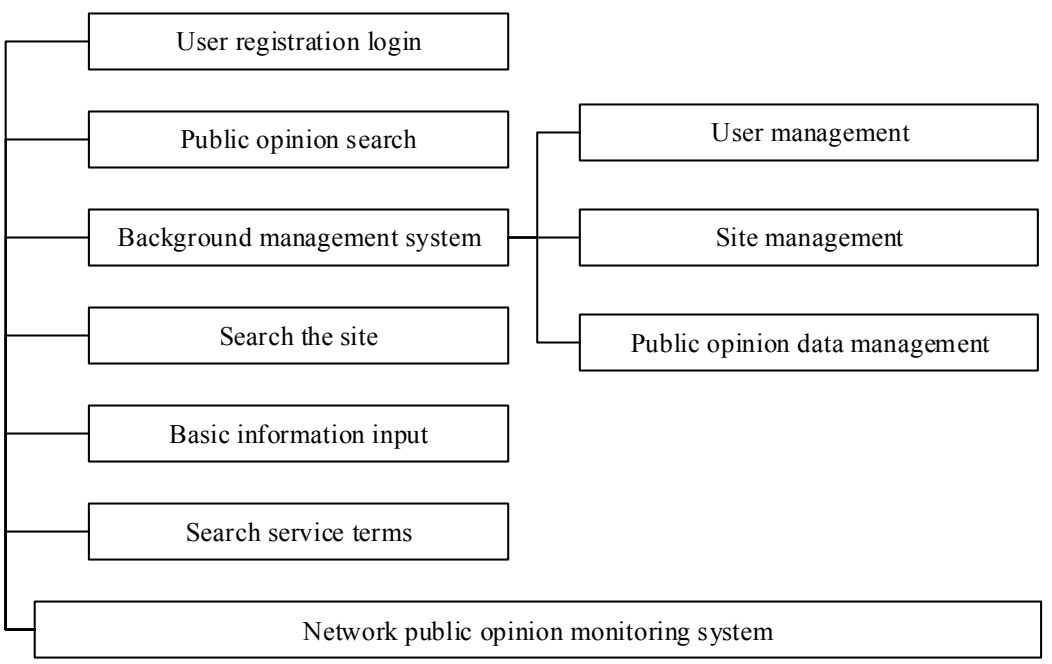

Fig. (2). Module structure design of the system. 


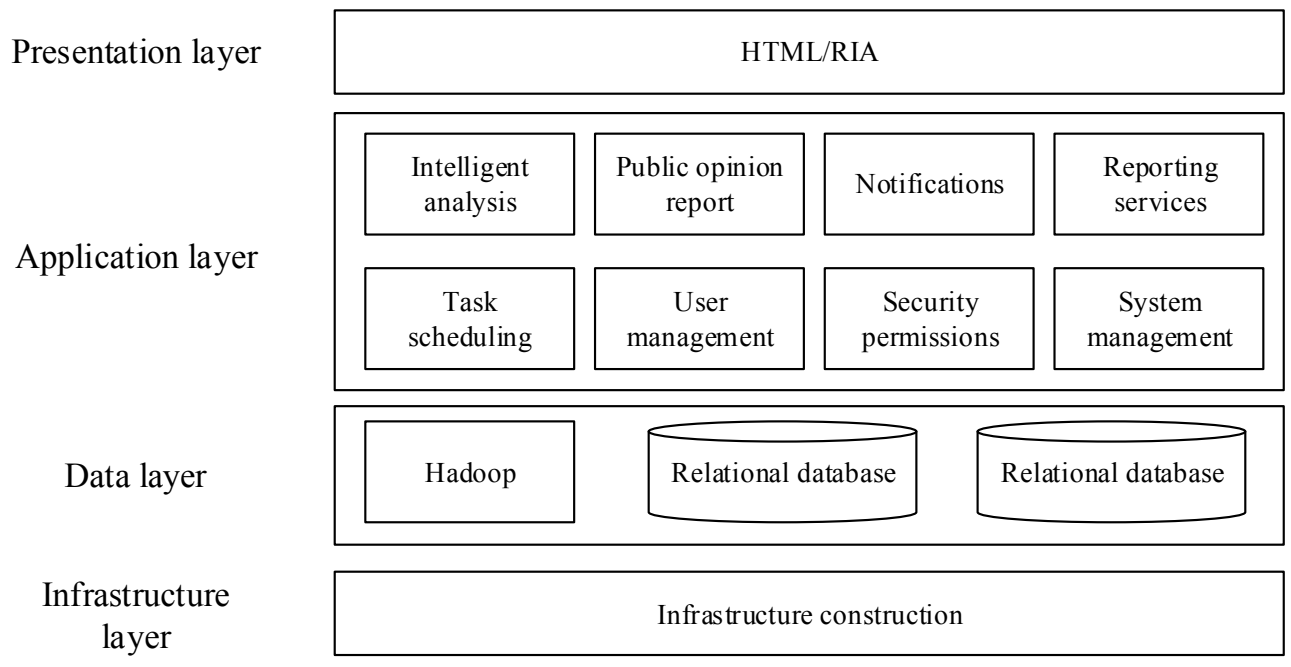

Fig. (3). System architecture of network public opinion monitoring management.

mation system. From the design level, it contains two aspects, the hardware system and software system, which consists of four servers and a message alarm. Four servers are respectively a Web server, a database server, a data processing server, and a data fetching server.

These four servers and SMS alarm function are as follows:

Web server. In charge of public opinion information presentation, undertaking negative expression conveys the user configuration and operation.

Database server. Responsible for storage of public opinion data information knowledge and system configuration information.

Data processing server. In charge of public opinion data processing and responsible for the operation of response to user requests.

Data fetching server. In charge of public opinion data fetching.

\section{CONCLUSION}

With the development of information technology, the highlight of innovative development is the increased speed of the computer and the Internet respectively. Especially in recent years, blog, microblogging, light blogging and social networking, and other forms of new network media, triggered a newer, faster, and bigger orders of magnitude of information explosion due to Internet freedom, providing a more convenient and more efficient platform to fully express their views. Also in accordance with the existing network, public opinion monitoring technology has put forward new challenges.

In this paper, the design and implementation of network public opinion monitoring system has been presented, which provides the overall solution ensuring the security and integrity of data, information management, and the urgent user request. It combines the public opinion requirements, searches for information, data processing, and manages public opinion disposal function. Performance and function of public opinion management system are analyzed so as to realize the demand to satisfy the area. Through this paper, we com- pleted the basic work flow of network public opinion management, which is convenient for users of public opinion and information management at the disposal of public opinion management system design and implementation, by improving the quality and efficiency of the public opinion management, and standardizing the operation of the public opinion information disposal procedures. System development complexity significantly reduced after the completion of the network public opinion management system, and we are more likely to continue to develop related work process, that can be successfully run in public opinion management system. The system has an intuitive interface, has a simple function and convenient operation, and in the development process, it reduces the user manpower and time, to achieve the goal of easy operation, easy maintenance, and durability.

\section{CONFLICT OF INTEREST}

The author confirms that this article content has no conflict of interest.

\section{ACKNOWLEDGEMENTS}

Declared none.

\section{REFERENCES}

[1] R.K.L. Ko, B.S. Lee, and S. Pearson, Towards Achieving Accountability, Auditability and Trust in Cloud Computing, Advances in Computing and Communications, Springer, Berlin Heidelberg, 2011, pp. 432-444

[2] R.K.L Ko, P. Jagadpramana, and M. Mowbray, "Trustcloud: a framework for accountability and trust in cloud computing", In: IEEE World Congress on Services (SERVICES), IEEE, 2011, pp. 584-588.

[3] K.P.N Puttaswamy, and B.Y. Zhao, "Preserving privacy in location-based mobile social applications" In: Proceedings of the $11^{\text {th }}$ Workshop on Mobile Computing Systems \& Applications. ACM, 2010, pp.1-6.

[4] A. Scalbert, L. Brennan, and O. Fiehn, "Mass-spectrometry-based metabolomics: limitations and recommendations for future progress with particular focus on nutrition research", Metabolomics, vol. 5 no. 4, pp. 435-458, 2009.

[5] S.Y. Kim, T.N.Van, and S.O Ogunlana, "Quantifying schedule risk in construction projects using Bayesian belief networks", International Journal of Project Management, vol. 2, no. 1, pp. 39-50, 2009. 
[6] B. Calder, J. Wang, and A. Ogus, "Windows azure storage: a highly available cloud storage service with strong consistency" In: Proceedings of the $23^{\text {rd }}$ ACM Symposium on Operating Systems Principles, ACM, 2011, pp.143-157.

[7] C. Xian-Yi11, Z. Ling-ling, and Z. Qian, "The framework of network public opinion monitoring and analyzing system based on semantic content identification", Journal of Convergence Information Technology, vol. 5, no. 10, pp. 1-5, 2010.
[8] B.S. McIntosh, J.C Ascough, and M. Twery, "Environmental decision support systems (EDSS) development-challenges and best practices", Environmental Modelling \& Software, vol. 26, no. 12, pp. 1389-1402, 2011.

[9] L. Xiaojun, "The design and implementation of internet public opinion monitoring and analyzing system, In: $2^{\text {nd }}$ International Conference on e-Business and Information System Security (EBISS)," IEEE, 2010, pp. 1-5.

Received: June 16, 2015

Revised: August 23, 2015

Accepted: September 11,2015

(C) Li Yan.; Licensee Bentham Open

This is an open access article licensed under the terms of the Creative Commons Attribution Non-Commercial License (http://creativecommons.org/ licenses/by-nc/4.0/) which permits unrestricted, non-commercial use, distribution and reproduction in any medium, provided the work is properly cited. 